\title{
Early Disseminated Mycobacterium Abscessus Complex Infection in an Infant with Coexisting Cystic Fibrosis and Progressive Familial Intrahepatic Cholestasis Case report and literature review
}

Sanjeewani A. Weerakoon, ${ }^{1}$ Maya Al Salti, ${ }^{2}$ Jalila Mohsin, ${ }^{1}$ Hilal Al Hashami, ${ }^{3}$ Tawfiq Al Lawati, ${ }^{3}$ Hussain Mohsin

ABSTRAC T: Mycobacterium abscessus complex (MABSC) is a rapidly growing mycobacterium and may rarely cause disseminated infections in immunocompromised patients. In patients with cystic fibrosis (CF), it peaks between the ages of 11 and 15 years. We present a five-month-old infant with coexisting CF and progressive familial intrahepatic cholestasis (PFIC) who had pulmonary and cutaneous dissemination of MABSC infection. The management of this disseminated infection in an infant with two coexisting chronic diseases was challenging and resulted in the rapid deterioration of lung function and progression of PFIC to liver cirrhosis with a fatal outcome.

Keywords: Cystic Fibrosis; Atypical Mycobacterium; Mycobacterium Abscessus Complex; Progressive Familial Intrahepatic Cholestasis; Case Report; Oman.

$\mathrm{M}$ YCOBACTERIUM ABSCESSUS COMPLEX (MABSC) belongs to a group of rapidly growing mycobacteria (RGM) that can be clinically significant. Diseases caused by RGM have been increasingly reported during the past few decades due to various factors including improvement of molecular diagnostic methods and the increase in population at risk for non-tuberculous mycobacterial (NTM) diseases. ${ }^{1}$ MABSC is implicated in causing a wide range of clinical diseases including localised skin and soft tissue infections and disseminated infections such as osteomyelitis, lymphadenitis, cutaneous, lung and bloodstream infections, particularly in an immunocompromised host. ${ }^{1-3}$

The presence of underlying chronic lung diseases predisposes an individual to pulmonary infections. ${ }^{2}$ NTM has been increasingly reported as a significant pathogen among cystic fibrosis (CF) patients and has been linked to poor prognosis among this group. ${ }^{4-7}$

Progressive familial intrahepatic cholestasis (PFIC) is an autosomal recessive liver disease that can present with early-onset progressive liver failure. ${ }^{8}$

Herein, we report a case of disseminated infection due to $M$. abscessus in an infant with coexisting CF and PFIC.

\section{Case Report}

A full-term male infant who has a 10-year-old sibling with CF, was referred to a tertiary care hospital at the age of two months with a chronic cough for one month and failure to thrive. He was diagnosed with CF based on elevated sweat chloride level $(>60$ $\mathrm{mmol} / \mathrm{L}$ ) and genetic testing that confirmed the presence of a homozygous $3120+1 \mathrm{G}>\mathrm{A}$ cystic fibrosis transmembrane conductance regulator (CFTR) gene mutation. The infant received intravenous antibiotics therapy and was started on pancreatic enzymes and fat-soluble vitamins. His throat swab culture was negative and he was discharged with an outpatient follow-up.

At the age of five months, the infant was admitted through the paediatric emergency department with a two-day history of fever, cough and difficulty in breathing. At the time of presentation, the infant was in respiratory failure with a respiratory rate of 70 breaths per minute, low oxygen saturation of $76 \%$ in room air, bilateral diffused crackles and wheezing. $\mathrm{He}$ required immediate tracheal intubation and mechanical ventilation. The infant looked icteric with a palpable liver $4-5 \mathrm{~cm}$ below the costal margin with no splenomegaly. Other systemic examinations were unremarkable.

The initial laboratory tests revealed a haemoglobin level of $12.1 \mathrm{~g} / \mathrm{dL}$ with leukocytosis (predominantly neutrophils) at $26.2 \times 10^{9}$ cells/L. He had elevated liver enzymes including alanine transaminase of 460 IU/L, aspartate aminotransferase of $768 \mathrm{IU} / \mathrm{L}$ and alkaline phosphatase of $316 \mathrm{IU} / \mathrm{L}$. The total bilirubin was $159 \mu \mathrm{mol} / \mathrm{L}$ (conjugated bilirubin at $121 \mu \mathrm{mol} / \mathrm{L}$ ) 

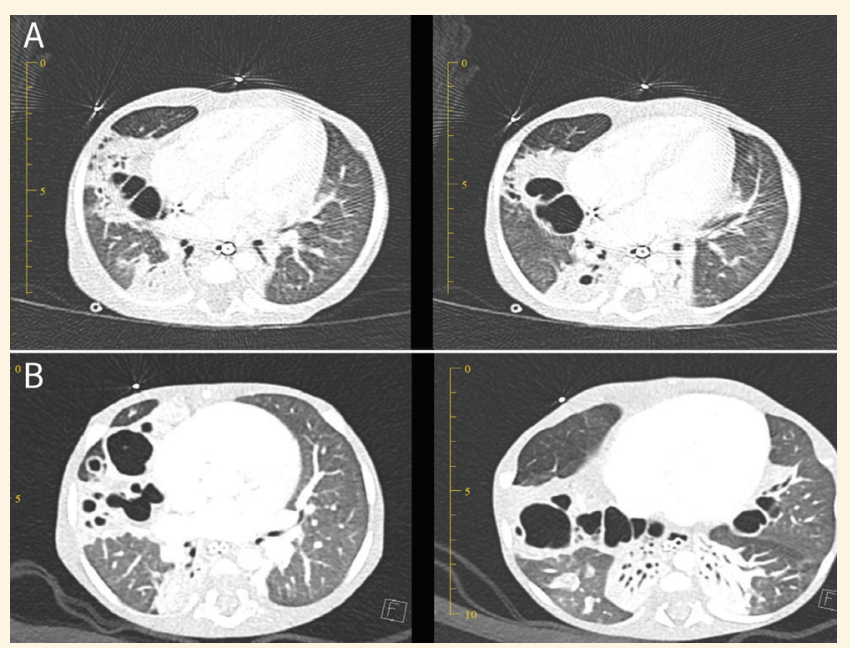

Figure 1: Computed tomography scans of the chest of a five-month old infant performed (A) before and (B) three months after the initiation of non-tuberculous mycobacterial treatment showing progressive bilateral cystic bronchiectasis.

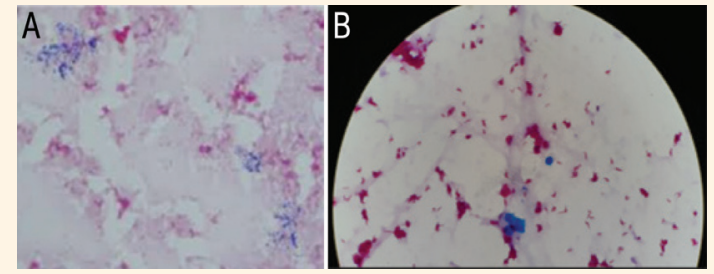

Figure 2: (A) Gram stain at $\times 100$ (oil immersion) magnification showing thin, beaded Gram-positive bacilli and (B) Ziehl-Neelson stain at $\times 100$ (oil immersion) magnification showing acid fast bacilli in a five-month old infant.

and serum albumin level was normal. The infant's coagulation profile was normal.

Chest X-ray showed hyperinflated lungs, peribronchial wall thickening and bilateral lower lobe consolidations. The infant was started on intravenous piperacillin-tazobactam $(90 \mathrm{mg} / \mathrm{kg} /$ dose four times daily), gentamicin (10 mg/kg/dose once daily) and oral oseltamivir (30 mg/dose twice daily) for the management of severe pneumonia and cystic fibrosis pulmonary exacerbation. Although the infant did not have a previously positive respiratory culture to guide antibiotic choice, his sibling was known to have chronic Pseudomonas aeruginosa airway infection. Hence, his sibling's chronic $P$. aeruginosa infection status was taken into consideration when choosing antibiotics to ensure adequate coverage.

During the first week of his stay at the paediatric intensive care unit (PICU), there was a deterioration in the infant's clinical condition, therefore the medication was changed from piperacillin-tazobactam to meropenem $(40 \mathrm{mg} / \mathrm{kg} /$ dose thrice daily). Initial blood cultures and respiratory samples (obtained via an endotracheal tube) for bacterial and fungal cultures and respiratory viral panel polymerase chain reaction $(\mathrm{PCR})$ were all negative. The infant continued to experience low-grade fever over the second week and had progressive worsening of his respiratory parameters which prompted performing a flexible bronchoscopy and obtaining bronchoalveolar lavage (BAL) fluid. The BAL sample was sent for bacterial, mycobacterium and fungal cultures and extended viral PCRs. The BAL sample microscopy showed a positive result for acid fast bacilli (AFB) through Ziehl-Neelson staining, while the tuberculosis PCR test done using GeneXpert (Cepheid, Sunnyvale, California, USA) was negative, raising the suspicion of NTM. A computed tomography (CT) scan of the chest showed bilateral cystic bronchiectasis and nodules [Figure 1].

Given the lack of improvement on broadspectrum antibiotics, the isolation of NTM from BAL and the CT changes, the child met the American Thoracic Society and the Infectious Diseases Society of America (ATS/IDSA) diagnostic criteria for nontuberculous mycobacterial pulmonary disease. ${ }^{6}$ On day 20 of admission, anti-tuberculous drugs-rifampicin (15 mg/kg/dose once daily), ethambutol (20 mg/kg/ dose once daily), clarithromycin $(7.5 \mathrm{mg} / \mathrm{kg} /$ dose twice daily) - were commenced while meropenem was continued.

The child developed a confluent macular-papular skin rash involving the face, trunk, limbs, palms and soles but sparing the mucus membranes. There was neither evidence of desquamation, erythroderma or angioedema clinically nor any blood eosinophilia. Disseminated cutaneous infection due to atypical mycobacteria was highly suspected after excluding other causes including drug-related hypersensitivity reaction. Around the same time, the AFB positive peripheral blood culture was also positive for Grampositive bacilli [Figure 2]. These were subsequently 
identified as $M$. abscessus by matrix-assisted laser desorption ionisation-time of flight mass spectrometry (MALDI-TOF MS). The central line blood culture remained negative for bacterial growth. The BAL culture was identified as $M$. abscessus through the line probe assay method using INNO-LiPA (LiPA Innogenetics, Ghent, Belgium). Azithromycin-based combination therapy with parenteral amikacin (18 $\mathrm{mg} / \mathrm{kg} /$ dose once daily) and meropenem (imipenem was not available) was started as an intensive phase regimen based on the US Cystic Fibrosis Foundation (CFF) and European Cystic Fibrosis Society (ECFS) recommendations for eight weeks. ${ }^{9}$ The isolate was not tested for standard drug sensitivity due to technical limitations. Subsequently, the treatment was switched to the continuation phase that included oral azithromycin (10 mg/kg/dose once daily), levofloxacin $(10 \mathrm{mg} / \mathrm{kg} /$ dose twice daily) and nebulised amikacin (250 mg/dose twice daily).

Furthermore, the infant had progressive cholestasis with elevated conjugated bilirubin and transaminitis. Based on a family history of PFIC in his first degree cousins, he underwent a liver biopsy which showed cholestatic hepatitis with fibrosis. A wholeexome sequence sample was sent to Centogene labs (Centogene N.V., Rostock, Germany), which found a homozygous mutation in the ATP binding cassette subfamily $B$ member 11 ( $A B C B 11$ ) gene, confirming the diagnosis of autosomal recessive PFIC type 2 in addition to CF. Given the early onset of NTM lung disease and the disseminated infection, the patient was extensively investigated for primary immunodeficiency disorders (PIDs) including Mendelian susceptibility to mycobacterial disease. However, no PIDs were found.

During his five-month stay in the hospital, the infant remained on respiratory support; mostly noninvasive ventilation with recurrent ICU admissions requiring intubation and mechanical ventilation. Multiple respiratory cultures obtained through the endotracheal tube and BAL remained negative for $M$. abscessus over the initial three months of treatment. However, a repeat BAL sample once again showed a positive result for M. abscessus in the fourth month of treatment. Two samples were positive for Aspergillus species with no other laboratory findings suggestive of allergic bronchopulmonary aspergillosis. The infant received four weeks of voriconazole $(10 \mathrm{mg} / \mathrm{kg} / \mathrm{dose}$ twice daily) that was switched to amphotericin B due to transaminitis. Despite being on ursodeoxycholic acid, his liver cirrhosis progressed and he developed portal hypertension.

Despite receiving anti-bacterial and antituberculous therapy and the supportive PICU care, the infant developed an acute-on-chronic deterioration of respiratory status. This led to rapid respiratory failure and death after five months of inpatient hospital stay.

\section{Discussion}

Disseminated infections due to $M$. abscessus are rare and occur mostly in immunocompromised patients. ${ }^{10,11}$ The current patient was investigated thoroughly for PIDs but none were found. To the best of the authors' knowledge, cases of coexisting conditions of CF and PFIC have never been reported before. Although CF is a known risk factor for NTM lung disease, it is not usually associated with disseminated NTM infection. Additionally, the onset of NTM lung disease in the infant was much earlier than what has been reported in the CF literature. ${ }^{12,13}$ PFIC type 2 typically results from a failure in the bile salt exporter pump (BSEP) protein which is responsible for exporting bile acids from the hepatocyte into canaliculi. This results in early-onset and progressive liver failure. ${ }^{8}$ Reviewing the literature, in general, PFIC or liver cirrhosis has not been shown to increase the susceptibility to NTM infection. It is possible that the coexistence of these two conditions resulted in a secondary immunodeficiency status which led to this disseminated infection. However, this conclusion cannot be made based on this single case.

The presence of at least one of the following characteristics would define the disseminated disease: cutaneous manifestations, involvement of more than one organ with or without cutaneous involvement, involvement of more than two groups of lymph nodes or positive blood culture. ${ }^{12}$ Cutaneous presentations can manifest as skin infections with discrete nodules, ulceration, multiple abscesses, diffuse maculopapular eruptions or, in contrast, as reactive skin lesions such as erythema nodosum, pustular psoriasis, generalised pustulosis and acute febrile neutrophilic dermatosis (Sweet syndrome). ${ }^{14}$ The current patient did have maculopapular skin eruptions; however, though the lesions were not biopsied, the patient showed dramatic improvement after the commencement of anti-NTM agents.

Lymphadenitis is the most common presentation of NTM in immunocompetent children. ${ }^{12}$ NTM infects patients with underlying chronic lung diseases such as bronchiectasis, emphysema and healed tuberculosis. It has emerged as a frequently isolated pathogen among CF patients over the past few decades with prevalence rates of up to $20 \%$ in USA and 13\% in Europe. ${ }^{4,5}$ According to the US CFF registry data (2016), M. abscessus was found to be the second most common isolate after $M$. avium complex accounting for $16 \%$ and $72 \%$ of positive cultures respectively. ${ }^{15}$ The isolation of $M$. abscessus from 
sputum culture does not necessarily indicate infection. Accurate differentiation of lung infection from lung colonisation is challenging but important since the infection contributes to a more fulminant disease outcome in CF patients. ${ }^{7}$ Furthermore, the recovery of M. abscessus from routine bacterial cultures is often masked and easily missed due to overgrowth of other common respiratory tract pathogens and colonising bacteria. In order to diagnose NTM lung disease, the following criteria should be fulfilled: clinical (persistent respiratory symptoms), radiological (nodular and/ or cavitary opacities on radiograph or bronchiectasis with nodules on CT scan) and microbiological evidence. ${ }^{6}$ Microbiological diagnostic criteria for NTM lung disease requires persistent growth of NTM in respiratory specimens: one positive AFB culture results in either two separate expectorated sputum samples or in one bronchial wash or lavage. ${ }^{6}$ The presence of a central venous catheter is a common predisposing factor for $M$. abscessus bloodstream infections. ${ }^{16}$ Identification of the NTM from blood culture may be difficult and often misdiagnosed with Corynebacterium spp. which are usually considered to be skin contaminants. ${ }^{16}$ This case highlights the importance of careful interpretation of Gram-positive bacilli which helps in the timely management of NTM disseminated infections.

Accurate and urgent identification of NTM down to the species level is important in deciding the correct antibiotic choice. Different laboratory methods are used for the identification of NTM including biochemical tests, high-performance liquid chromatography and molecular tests. ${ }^{6,9}$ A reverse hybridisation assay such as 'INNO-LiPA Mycobacteria v2' (LiPA Innogenetics) is designed to amplify the mycobacterial $16 \mathrm{~S}-23 \mathrm{~S}$ rRNA internal transcribed spacer region. However, it cannot differentiate $M$. abscessus on a sub-species level. ${ }^{9,17}$ A recent study shows that MALDI-TOF MS can be used with promising results to identify NTM species including $M$. abscessus (concordance results with a reverse hybridisation-based assay showing 96.9\% agreement). ${ }^{18}$

Management of pulmonary and extra-pulmonary MABSC infection is faced with multiple challenges including intrinsic resistance to the common, standard anti-tuberculous agents, discordant in vitro susceptibility and clinical response, limited provento-be-effective drugs, serious side-effects and the long duration required for clinical and microbiological cure. $^{19}$ To overcome these difficulties, a multidisciplinary team which includes a chest physician, infectious disease expertise, clinical microbiologist, clinical pharmacist, physiotherapist, community nurse, the patient and his/her family is needed. ${ }^{20}$
The ATS/IDSA 2007 guideline recommends a multidrug, macrolide-based regimen along with surgical resection, if possible, for both pulmonary and extrapulmonary infections. ${ }^{6}$ As per the US CFF and ECFS consensus recommendations, the treatment is divided into an initial intensive phase (which can range from three to 12 weeks) followed by a continuation phase. ${ }^{9}$ The intensive phase includes an oral macrolide, intravenous amikacin and one or more of the following: intravenous tigecycline, imipenem or cefoxitin. The decision to switch to the continuation phase should depend on the response to treatment, the severity of infection and if the regimen is tolerated by the patient. The continuation phase includes an oral macrolide, inhaled amikacin and two to three out of the following antibiotics: minocycline, clofazimine, moxifloxacin and linezolid. ${ }^{9}$ Antibiotic choices should be guided but not dictated by drug susceptibility testing. ${ }^{17}$ Inducible resistance to clarithromycin is another challenge and it is due to the presence of the erythromycin ribosome methyltransferase (erm) gene in two of the $M$. abscessus sub-species: $M$ abscessus subsp. abscessus and M. abscessus subsp. bolletii. In comparison, M. abscessus subsp. massiliense does not have inducible resistance to clarithromycin due to the non-functioning erm gene, making therapeutic response to macrolide-based therapy more effective and efficient., ${ }^{2,21,22}$ Unavailability of recently validated techniques such as multi-locus sequence typing of hsp65, rрoB and secA genes for differentiation into sub-species levels, was an additional limitation in optimising antibiotic treatment in our case. ${ }^{23}$

\section{Conclusion}

The current case report describes a fatal case of disseminated $M$. abscessus infection in an infant who had two chronic comorbid conditions of CF and PFIC along with the diagnostic and therapeutic challenges associated with case management. This report highlights the importance of having a high index of suspicion for NTM infection in children with CF presenting with early deterioration of lung function. More research with regard to the optimal management of NTM infection in this young population is required.

\section{AUTHORS' CONTRIBUTION}

SW and MAS drafted the initial manuscript focusing on the microbiology aspect. HM added the clinical presentation and discussion sections. HAH reviewed the manuscript and updated the infectious disease aspect. JM reviewed and updated the microbiology aspect. TAL wrote and reviewed the liver disease aspects. All authors approved the final version of the manuscript. 


\section{References}

1. Brown-Elliot BA, Wallace Jr RJ. Infections Due to Nontuberculous Mycobacteria Other than Mycobacterium avium-intracelluare. In Mandell GL, Bennett JE, Dolin R, Eds. Mandell, Douglas and Bennet's Principles and Practice of Infectious Diseases. 7th ed. Philadelphia: Elsevier, 2010. Pp. 3191-8.

2. De Groote MA, Huitt G. Infections due to rapidly growing mycobacteria. Clin Infect Dis 2006; 42:1756-63. https://doi.org/10.10 $86 / 504381$.

3. Sfeir M, Walsh M, Rosa R, Aragon L, Liu SY, Cleary T, et al. Mycobacterium abscessus Complex Infections: A Retrospective Cohort Study. Open Forum Infect Dis 2018; 5:ofy022. https:// doi.org/10.1093/ofid/ofy022.

4. Cystic Fibrosis Foundation. Cystic Fibrosis Foundation Patient Registry 2016 Annual Data Report. From: https://prod-test.cff. org/Research/Researcher-Resources/Patient-Registry/2016Patient-Registry-Annual-Data-Report.pdf Accessed: Apr 2021.

5. Valenza G, Tappe D, Turnwald D, Frosch M, König C, Hebestreit H, et al. Prevalence and antimicrobial susceptibility of microorganisms isolated from sputa of patients with cystic fibrosis. J Cyst Fibros 2008; 7:123-7. https://doi.org/10.1016/j.jcf.2007.06.006.

6. Griffith DE, Aksamit T, Brown-Elliott BA, Catanzaro A, Daley C, Gordin F, et al. An official ATS/IDSA statement: Diagnosis, treatment, and prevention of nontuberculous mycobacterial diseases. Am J Respir Crit Care Med 2007; 175:367-416. https://doi. org/10.1164/rccm.200604-571ST.

7. Esther CR Jr, Henry MM, Molina PL, Leigh MW. Nontuberculous mycobacterial infection in young children with cystic fibrosis. Pediatr Pulmonol 2005; 40:39-44. https://doi.org/10.10 02/ppul.20222.

8. Srivastava A. Progressive Familial Intrahepatic Cholestasis. J Clin Exp Hepatol 2014; 4:25-36. https://doi.org/10.1016/j.jceh.20 13.10.005

9. Floto RA, Olivier KN, Saiman L, Daley CL, Herrmann JL, NickJA, et al. US Cystic Fibrosis Foundation and European Cystic Fibrosis Society consensus recommendations for the management of non-tuberculous mycobacteria in individuals with cystic fibrosis. Thorax 2016;71:i1-i22. https://doi.org/10.1136/thoraxjnl-2015-207360.

10. Gardner AI, McClenaghan E, Saint G, McNamara PS, Brodlie M Thomas MF. Epidemiology of Nontuberculous Mycobacteria Infection in Children and Young People With Cystic Fibrosis: Analysis of UK Cystic Fibrosis Registry. Clin Infect Dis 2019; 68:731-7. https://doi.org/10.1093/cid/ciy531.

11. Zimmermann P, Curtis N, Tebruegge M. Nontuberculous mycobacterial disease in childhood - update on diagnostic approaches and treatment. J Infect. 2017 Jun; 74:S136-42. https://doi. org/10.1016/S0163-4453(17)30204-9.

12. Chetchotisakd P, Mootsikapun P, Anunnatsiri S, Jirarattanapochai $\mathrm{K}$, Choonhakarn C, Chaiprasert A, et al. Disseminated infection due to rapidly growing mycobacteria in immunocompetent hosts presenting with chronic lymphadenopathy: A previously unrecognized clinical entity. Clin Infect Dis 2000; 30:29-34. https:// doi.org/10.1086/313589.
13. Roux AL, Catherinot E, Ripoll F, Soismier N, Macheras E, Ravilly S, et al. Multicenter study of prevalence of nontuberculous mycobacteria in patients with cystic fibrosis in france. J Clin Microbiol 2009; 47:4124-8. https://doi.org/10.1128/JCM.01257-09.

14. Lee MR, Sheng WH, Hung CC, Yu CJ, Lee LN, Hsueh PR. Mycobacterium abscessus Complex Infections in Humans. Emerg Infect Dis 2015; 21:1638-46. https://doi.org/10.320 $1 / 2109.141634$.

15. Olivier KN, Weber DJ, Wallace RJ Jr, Faiz AR, Lee JH, Zhang Y, et al. Nontuberculous mycobacteria. I: Multicenter prevalence study in cystic fibrosis. Am J Respir Crit Care Med 2003; 167:828-34. https://doi.org/10.1164/rccm.200207-678OC.

16. El Helou G, Viola GM, Hachem R, Han XY, Raad II. Rapidly growing mycobacterial bloodstream infections. Lancet Infect Dis 2013; 13:166-74. https://doi.org/10.1016/S1473-3099(12)7 0316-X.

17. Suffys PN, da Silva Rocha A, de Oliveira M, Campos CE, Barreto AM, Portaels F, et al. Rapid identification of Mycobacteria to the species level using INNO-LiPA Mycobacteria, a reverse hybridization assay. J Clin Microbiol 2001; 39:4477-82. https:// doi.org/10.1128/JCM.39.12.4477-4482.2001.

18. Mediavilla-Gradolph MC, De Toro-Peinado I, Bermúdez-Ruiz MP, García-Martínez Mde L, Ortega-Torres M, Montiel QuezelGuerraz N, et al. Use of MALDI-TOF MS for Identification of Nontuberculous Mycobacterium Species Isolated from Clinical Specimens. Biomed Res Int 2015; 2015:854078. https://doi. org/10.1155/2015/854078.

19. Tippett E, Ellis S, Wilson J, Kotsimbos T, Spelman D. Mycobacterium abscessus complex: Natural history and treatment outcomes at a tertiary adult cystic fibrosis center. Int J Mycobacteriol 2018; 7:109-16. https://doi.org/10.4103/ijmy.ijmy_55_18.

20. Harris KA, Kenna DTD. Mycobacterium abscessus infection in cystic fibrosis: molecular typing and clinical outcomes. J Med Microbiol 2014; 63:1241-6. https://doi.org/10.1099/jmm.0.077164-0.

21. Zelazny AM, Root JM, Shea YR, Colombo RE, Shamputa IC, Stock F, et al. Cohort study of molecular identification and typing of Mycobacterium abscessus, Mycobacterium massiliense, and Mycobacterium bolletii. J Clin Microbiol 2009; 47:1985-95. https://doi.org/10.1128/JCM.01688-08.

22. Rubio M, March F, Garrigó M, Moreno C, Español M, Coll P. Inducible and Acquired Clarithromycin Resistance in the Mycobacterium abscessus Complex. PLoS One 2015; 10:e0140166. https://doi.org/10.1371/journal.pone.0140166.

23. M Macheras E, Konjek J, Roux AL, Thiberge JM, Bastian S, Leão SC, et al. Multilocus sequence typing scheme for the Mycobacterium abscessus complex. Res Microbiol 2014; 165:82-90. https://doi.org/10.1016/j.resmic.2013.12.003. 